\title{
Hepatic sclerosing hemangioma mimics hepatic malignancies: a case report and multidisciplinary approach
}

\author{
Dina M. Sweed ${ }^{1 *}$ D, Zeinab A. Fayed ${ }^{1}$, Enas M. Sweed ${ }^{2}$, Ahmed El-Sherif ${ }^{3}$ and Mohamed Mohamady ${ }^{1}$
}

\begin{abstract}
Background: Hepatic sclerosing hemangiomas $(\mathrm{HSH})$ are rare benign vascular tumors that mimic radiologically hepatic malignancies. HSH is characterized by extensive fibrosis and hyalinosis as a result of degeneration and thrombosis. Pre-operative diagnosis is very difficult and most of the cases undergo surgical resections.

Case presentation: A 65 years old, hepatitis C virus-infected female presented by an accidentally discovered hepatic focal lesion. Magnetic resonance imaging (MRI) showed a T2 hyperintense mass displaying peripheral enhancement in porto-venous phase. The patient had a simultaneous typical hepatic hemangioma and multiple vertebral bodies' hemangiomata. Tissue-guided biopsy revealed a densely sclerotic stroma containing vascular spaces with occasional obliteration of the lumen. Immunohistochemical staining was performed for Pan CK, CD34, SMA, VEGF, and c-Kit which confirmed the vascular nature of neoplasm and the involuting phase of hemangioma development. The patient was reassured and recommended for a regular radiological follow-up to reassess the lesion size.
\end{abstract}

Conclusion: The definitive preoperative diagnosis of HSH is still problematic. However, the awareness of surgeons by this entity, peripheral enhancement in porto-venous phase on dynamic MRI, the presence of simultaneous typical hepatic hemangioma in the absence of cancer history could raise suspicion for HSH. Liver biopsy is still the gold standard in approaching the accurate diagnosis.

Keywords: Biopsy, Hemangioma, MRI, Sclerosing

\section{Background}

Hepatic hemangioma is the most common benign tumor occurring in the liver. Hemangioma is a vascular tumor that passes through three stages of development: angiogenesis, regression, and involution [1]. The diagnosis is generally secured on radiologic examination with magnetic resonance imaging (MRI) is the most sensitive and specific imaging tool [2]. However, the sclerosing variant of hepatic hemangioma is extremely rare and mimics radiologically hepatic malignancies which necessitate surgical intervention [3, 4]. Here, we report a case of hepatic sclerosing hemangioma $(\mathrm{HSH})$ that simulates liver metastasis radiologically and give a short brief on the pathogenesis of this rare variant.

\footnotetext{
* Correspondence: dr.dinasweed@yahoo.com

${ }^{1}$ Pathology Department, National Liver Institute, Menoufia University, Al

Menoufia, Egypt

Full list of author information is available at the end of the article
}

\section{Case presentation}

A 65-year-old female presented to a medical physician when hepatic focal lesion during routine examination for hepatitis $\mathrm{C}$ virus $(\mathrm{HCV})$ infection was accidentally discovered. The patient was diabetic, hypertensive, and obese. There was no past or present history of cancer. She was HCV positive. Serum alpha-fetoprotein (AFP) level was 6.1 IU/mL, CA19-9 42.6 U/L, and CEA $2.7 \mathrm{ng} /$ $\mathrm{mL}$. Laboratory investigations were normal apart of slight anemia (hemoglobin $12 \mathrm{~g} / \mathrm{dL}$ ). Clinical and radiological findings established the primary hepatic origin of the lesion with no extrahepatic masses. Imaging showed a left hepatic lobe segment II focal lesion measured $3.5 \times 3 \mathrm{~cm}$ surrounded by focal intrahepatic biliary radicles dilatation (IHBRD) on a background of noncirrhotic liver. The mass showed a rather well defined hyperintense in axial fat suppression T2 with peripheral enhancement in porto-venous phase and hypointense 
with filling defect in the hepatocyte (late) phase of dynamic MRI. A simultaneous left hepatic lobe segment $\mathrm{IVb}$ focal lesion measuring $1.5 \mathrm{~cm}$ showed a rather well defined hyperintense with discontinuous peripheral nodular enhancement in arterial phase with central fill in in the delayed phase (Fig. 1). Osseous vertebral bodies' hemangiomata were accidentally discovered. The radiological features were in favor of hepatic metastasis or intrahepatic cholangiocarcinoma (IHCC). However, the rare possibility of HSH could not be ruled out for liver-guided biopsy.

Histopathological examination showed thin-walled vascular spaces lined by bland-looking endothelial cells within a hypocellular stroma demonstrating varying degrees of fibrosis and sclerosis. Some blood vessels were obliterated. Focal area of the classic cavernous hemangioma was evident. The dense sclerotic stroma was demarcated by masson trichrome (MT) stain. The adjacent liver tissue showed features of chronic hepatitis consistent with $\mathrm{HCV}$ infection. Immunohistochemical studies (IHC) for CD34 confirmed the vascular nature of neoplastic cells. The negative staining for cytokeratin (CK) marker ruled out the rare possibility of biliary hamartoma. In an attempt to define the phase of development of hemangioma, a panel of IHC stain was performed. Vascular spaces showed immunopositivity for smooth muscle actin (SMA) and negativity for vascular endothelial growth factor (VEGF). Additionally, c-Kit
IHC highlighted degenerated mast cells granules (Fig. 2). The patient was reassured and discharged on a regular radiological follow up to re-evaluate the mass size.

\section{Discussion}

Hepatic sclerosing/sclerosed hemangioma ( $\mathrm{HSH})$ is a rare benign vascular tumor showing degenerative changes, fibrosis, and sclerosis as a result of thrombosis of the blood vessels [5]. It was first described by Shepherd and Lee as a possible cause of solitary necrotic hepatic nodules [6]. Berry reported another two cases of accidentally discovered HSH in a study of 1000 autopsy specimens [7]. In 1994, the objective basis for the development of HSH was postulated, and the lesion was recognized as a mimicker of hepatic malignancy [1]. Yugawa et al. reported the characterization of 41 cases of HSH [8]. However, after a thorough search for the terms of sclerosing, sclerosed, or hyalinized, we recognized 77 cases in the literatures including the present case. The clinico-radiological and histopathological features were summarized in Table 1 . The median age was in sixth or seventh decades with female predominance. Nine cases raised on top of chronic liver disease; HBV or $\mathrm{HCV}$, non-alcoholic fatty liver disease (NAFLD), or mixed HBV and alcoholic liver disease. In addition, seven cases had metabolic syndrome; diabetes mellitus, obesity, or hyperlipidemia. The present case was female, diabetic, and obese accidentally discovered HCV infection. The majority

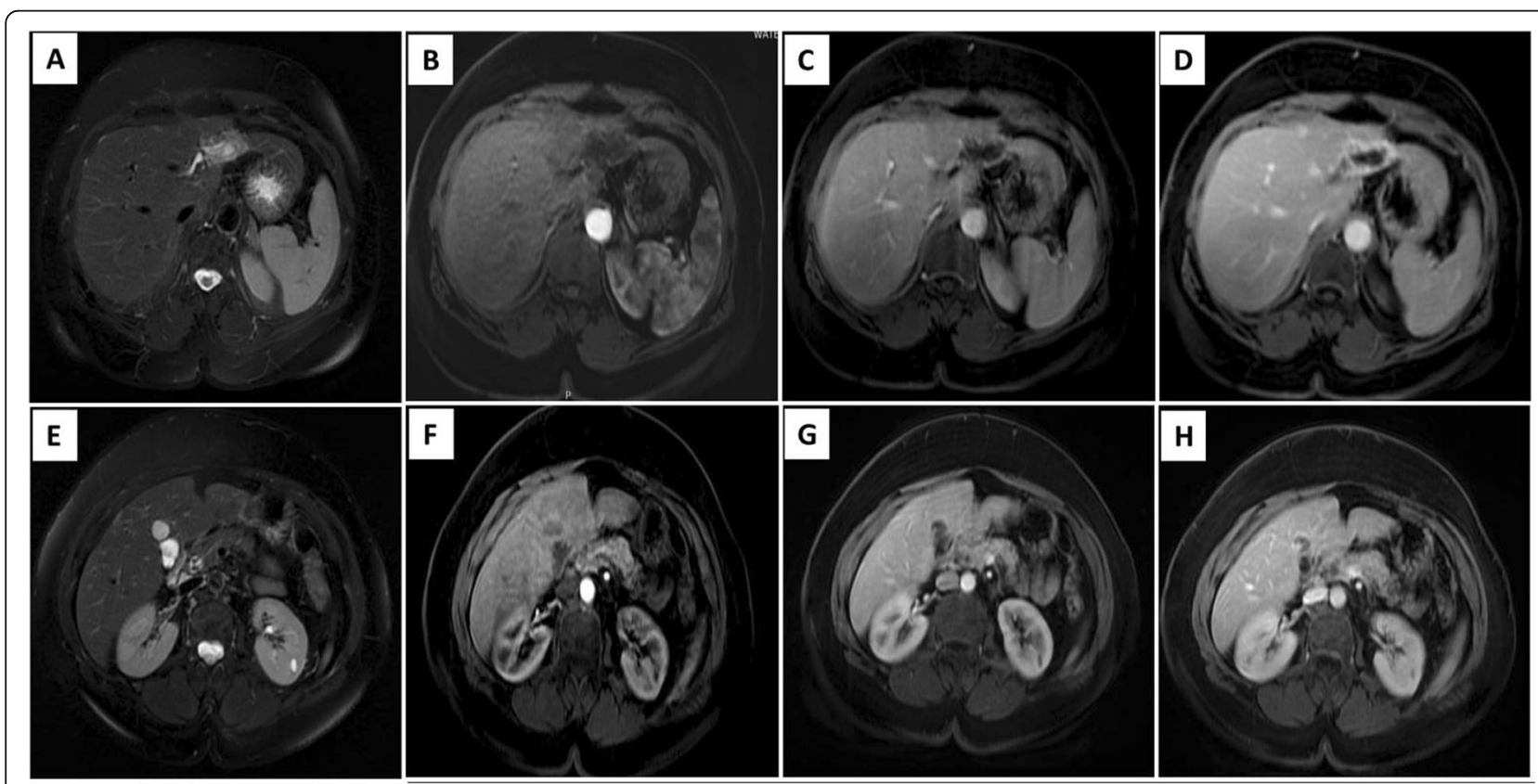

Fig. 1 a Axial fat suppression T2 showed a rather well-defined hyperintense left hepatic lobe segment II focal lesion surrounded by focal intrahepatic biliary radicles dilatation on background of non-cirrhotic liver. $\mathbf{b}$ Inconscipous enhacenemt in arterial phase $\mathbf{c}$ Mild ring enhancement in porto-venous and $\mathbf{d}$ Obvious ring enhacement in delayed phase of dynamic MRI. e Axial T2 fat suppression of the typical hemangioma showwd a rather well-defined bright left hepatic lobe segment IVb focal lesion, $\mathbf{f}$ Discontinuous peripheral nodular enhancement in arterial phase, $\mathbf{g}$ Centripetal fillin in porto-venous and $\mathbf{h}$ Closed iris sign in delayed equilibrium phase 


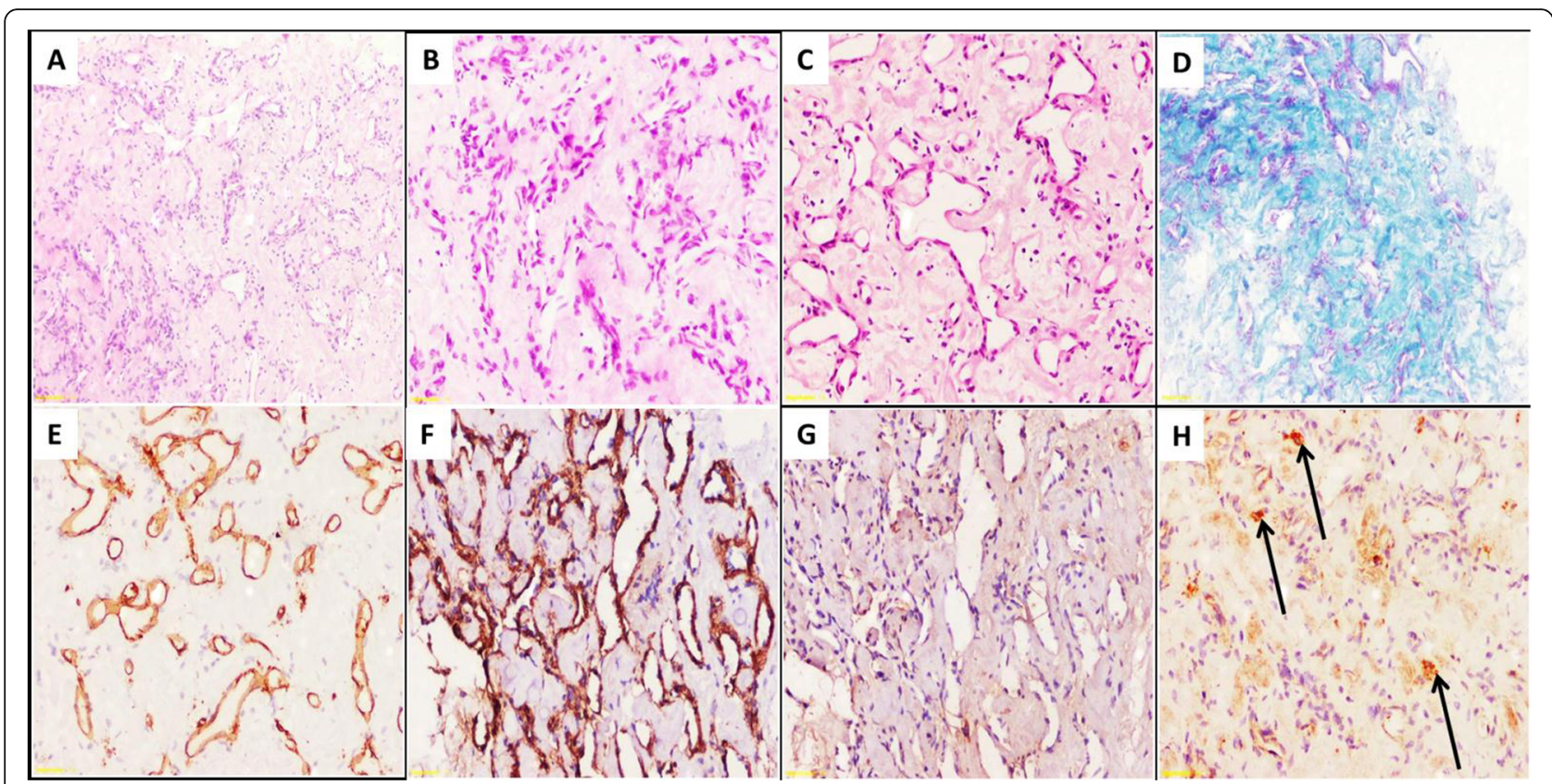

Fig. 2 a A case of HSH showed proliferated, focally obliterated blood vessels surrounded by dense sclerotic stroma (H\&E 100x). b Higher power view (H\&E 200X). c Area of cavernous hemangioma (H\&E 200X). d Dense sclerotic stroma (MT 100X). e Vascular spaces were positive for CD34 (IHC 200x). f Smooth muscle proliferation was positive for SMA (IHC 200x). g Endothelial cells and fibroblasts were negative for VEGF (IHC 200x). h Degenerated masts cells were positive for C-Kit (IHC 200x)

of $\mathrm{HSH}$ was discovered during abdominal imaging or for abdominal pain [9]. However, only one case of ruptured sclerosing hemangioma was reported [10]. Nearly $78 \%$ and $60 \%$ of cases were solitary and located in the right lobe of the liver, respectively.

Typical hepatic hemangioma has a diagnostic peripheral nodular discontinuous enhancement following a blood pool on computed tomography (CT) with the MRI has the highest sensitivity and specificity [2]. However, the preoperative radiological diagnosis of $\mathrm{HSH}$ is very difficult and mimics hepatic malignancies; 23 cases, 9 cases, and 7 cases were misdiagnosed as metastasis, hepatocellular carcinoma (HCC), and IHCC, respectively. In the present case, the liver was non-cirrhotic with the lesion did not follow the typical pathological enhancement pattern of HCC, homogenous arterial enhancement, washout of contrast in porto-venous, and hypointense in hepatocyte phase of dynamic MRI study. The presence of focal IHBRD was in favor of IHCC but the absence of delayed enhancement made this diagnosis unlikely. Additionally, IHBRD may occur as a consequence of all intra- or extra-hepatic lesions with mass effect especially around segment IV [11]. The obvious peripheral enhancement of the lesion in the portovenous phase favored metastatic process that should be considered at the top of differential diagnosis. However, the patient had no past or present history of any cancer.
The awareness of radiologist for the radiological features of $\mathrm{HSH}$, hyperintense $\mathrm{T} 2$, and the presence of typical hepatic hemangioma and vertebral column hemangiomata raised the suspicious of HSH. With advanced radiological imaging, Miyata et al. postulated that apparent diffusion coefficient (ADC) mean to differentiate $\mathrm{HSH}$ from other liver malignancies as, in particular, the higher ADC mean value of using diffusion-weighted sequences (DW-MRI). They reported the ADC mean value of $\mathrm{HSH}$ was $1.94 \times 10-3 \mathrm{~mm} 2 / \mathrm{s}$, which was higher than the value of common malignant liver tumors [4]. In the present case, liver biopsy and pathological review were advised to elucidate the nature of the lesion.

Tissue pathology was the standard tool to establish the diagnosis. Tissue was obtained at autopsy in 5 cases, surgical resection specimen in 37 cases and liver biopsy in 13 cases. Only two cases were proofed radiological to be HSH on regular follow up $[12,13]$. In the present cases, liver core biopsy was obtained and assessed by two independent liver pathologists. In routine hematoxylin and eosin stain, dense acellular fibrous tissue with few dispersed blood vessels lined by flattened endothelial cells was evident. There was no evidence of cytological atypia, mitosis, or necrosis. Perivascular smooth muscle proliferation was occasionally seen. MT stain established dense fibrous stroma. IHC staining was not essential except in the area of dense sclerosis to rule out the rare 
Table 1 Review of literature for hepatic sclerosing hemangioma (HSH) cases

\begin{tabular}{|c|c|}
\hline Variables & No (\%) \\
\hline Median age & 65.5 years \\
\hline Gender (M:F ratio) & $31 / 45$ \\
\hline \multicolumn{2}{|l|}{ Site } \\
\hline Right & $36(60 \%)$ \\
\hline Left & $19(31.7 \%)$ \\
\hline Both lobes & $5(8.3 \%)$ \\
\hline \multicolumn{2}{|l|}{ Focality } \\
\hline Solitary & $40(78.4 \%)$ \\
\hline Multiple & $11(21.6 \%)$ \\
\hline Size & $48.2 \pm 45 \mathrm{~mm}$ \\
\hline \multicolumn{2}{|l|}{ CT findings } \\
\hline Ring enhancement & $39(73.6 \%)$ \\
\hline No enhancement & $6(11.3 \%)$ \\
\hline Heterogeneous or intralesional enhancement & $5(9.4 \%)$ \\
\hline Peripheral nodular enhancement & $3(5.7 \%)$ \\
\hline \multicolumn{2}{|l|}{ MRI (T1/T2) } \\
\hline Low/high & $40(93.2 \%)$ \\
\hline High/high & $2(4.5 \%)$ \\
\hline Low/low & $1(2.3 \%)$ \\
\hline \multicolumn{2}{|l|}{ Diagnostic confirmation } \\
\hline Surgery & $37(64.9 \%)$ \\
\hline Biopsy & $13(22.8 \%)$ \\
\hline Autopsy & $5(8.8 \%)$ \\
\hline Radiology & $2(3.5 \%)$ \\
\hline
\end{tabular}

M:F male:female, $C T$ computed tomography, MRI magnetic resonance imaging

possibility of biliary lesion. The neoplastic cells were positive for CD34 and negative for CK7 confirm the vascular nature of neoplasm.

There is no consensus, however, as to the state of involution of hemangiomas. Several theories have been proposed. Takahashi et al. reported the role of VEGF in promoting angiogenesis and endothelial proliferation in the proliferating phase [1]. On the contrary, SMA was expressed in the pericytes and functioned through inhibition of endothelial cell proliferation in the involuting and involuted phases. Makhlouf and Ishak found high immunopositivity for CD34 and SMA in sclerosing rather than sclerosed hemangioma and the role of mast cells in the induction of angiogenesis, regression, and fibrogenesis [14]. In addition, Choi et al. reported a case of sclerosing hemangioma with predominant pericapillary smooth muscle proliferation [15]. Therefore, we performed a panel of IHC to estimate the possible phase of hemangioma. The endothelial cells were negative for VEGF indicating the arrested phase of proliferation. SMA highlighted perivascular smooth muscle proliferation and
c-Kit was positive in the degenerated mast cells. These findings were inconsistent with the sclerosing phase of hemangioma.

\section{Conclusion}

Despite $\mathrm{HSH}$ are benign tumors that remain stable or even regressed over time, resection is necessary for large size lesions. The awareness of the imaging characteristics of HSH can alter patient management. Preoperative tissue biopsy or intraoperative frozen section should be considered to avoid unnecessary extended hepatic resection. However, if tumor malignancy cannot be ruled out or if the biopsy is hemorrhagic, hepatic resection should remain the choice for diagnostic surgery at present.

\section{Abbreviations \\ ADC: Apparent diffusion coefficient; AFP: Alpha foetoprotein; CD34: Cluster of differentiation 34; CK: Cytokeratin; CT: Computed tomography; DW: Diffusion- weighted; HBV: Hepatitis B virus; HCC: Hepatocellular carcinoma; HCV: Hepatitis C virus; HSH: Hepatic sclerosing hemangioma; IHBRD: Intrahepatic biliary radicles dilatation; IHC: Immunohistochemical studies; IHCC: Intrahepatic cholangiocarcinoma; MRI: Magnetic resonance imaging; MT: Masson trichrome; NAFLD: Non-alcoholic fatty liver disease; SMA: Smooth muscle actin; VEGF: Vascular endothelial growth factor \\ Acknowledgements \\ Not applicable.

\begin{abstract}
Authors' contributions
All authors of this paper have participated in its drafting and approved the final version submitted. DS, ZF, and ES wrote the case report. DS and ZF contributed to the pathological diagnosis. ES offered assistance for radiological diagnosis. MM performed technical immunohistochemical work. AS acquired and interpreted the clinical data. DS revised and edited the final version. All authors read and approved the final manuscript.
\end{abstract}

Funding

Not applicable.

Availability of data and materials

The availability of data and materials are available from the corresponding author on a reasonable request.

Ethics approval and consent to participate

The study was in accordance with an approved Institutional Review Board (IRB) protocol of the National Liver Institute (NLI), Menoufia University.

\section{Consent for publication}

Written informed consent was obtained from the patient for publication of this case report and accompanying images.

\section{Competing interests}

The authors declare that they have no competing interests.

\section{Author details}

'Pathology Department, National Liver Institute, Menoufia University, Al Menoufia, Egypt. ${ }^{2}$ Radiology Department, Faculty of Medicine, Benha University, Benha, Egypt. ${ }^{3}$ Hepato-pancreatobiliary Surgery and Liver Transplantation, National Liver Institute, Menoufia University, Al Menoufia, Egypt. 
Received: 30 July 2019 Accepted: 14 October 2019

Published online: 14 November 2019

References

1. Takahashi K, Mulliken JB, Kozakewich HP, Rogers RA, Folkman J, Ezekowitz RA (1994) Cellular markers that distinguish the phases of hemangioma during infancy and childhood. J Clin Invest 93(6):2357-2364

2. Taseva A, Tasev V, Bulanov D, Dimitrov K, Popov V, Zivkov E et al (2013) Diagnosis of liver hemangioma. Khirurgiia (Sofiia) (3):8-13

3. Sugo H, Sekine Y, Miyano S, Watanobe I, Machida M, Kojima K et al (2018) Hepatic sclerosing hemangioma with predominance of the sclerosed area mimicking a biliary cystadenocarcinoma. Case Reports Hepatol 2018:6

4. Miyata T, Beppu T, Kuramoto K, Nakagawa S, Imai K, Hashimoto D et al (2018) Hepatic sclerosed hemangioma with special attention to diffusionweighted magnetic resonance imaging. Surg Case Rep 4(1):3

5. Song JS, Kim YN, Moon WS (2013) A sclerosing hemangioma of the liver. Clin Mol Hepatol 19(4):426-430

6. Shepherd NA, Lee G (1983) Solitary necrotic nodules of the liver simulating hepatic metastases. J Clin Pathol 36(10):1181-1183

7. Berry CL (1985) Solitary "necrotic nodule" of the liver: a probable pathogenesis. J Clin Pathol 38:1278-1280

8. Yugawa K, Yoshizumi T, Harada N, Motomura T, Harimoto N, Itoh S et al (2018) Multiple hepatic sclerosing hemangiomas: a case report and review of the literature. Surg Case Rep 4(1):60

9. Caseiro-Alves F, Brito J, Araujo AE, Belo-Soares P, Rodrigues H, Cipriano A et al (2007 Jun) Liver haemangioma: common and uncommon findings and how to improve the differential diagnosis. Eur Radiol 17(6):1544-1554

10. Papafragkakis H, Moehlen M, Garcia-Buitrago MT, Madrazo B, Island E, Martin P (2011) A case of a ruptured sclerosing liver hemangioma. Int J Hepatol 2011:942360

11. Yang DM, Kim HS, Cho SW (2002) Pictorial review: various causes of hepatic capsular retraction: CT and MR findings. Br J Radiol 75(900):994-1002

12. Shin YM (2011) Sclerosing hemangioma in the liver. Korean J Hepatol 17(3): 242-246

13. Nunes LMAO, CDd M-A, Yamauchi Fl, Baroni RH (2018) Atypical hepatic hemangioma: imaging features of hyalinized hemangioma. Einstein (Sao Paulo, Brazil) 16(2):eAl4256-eAl4eAl

14. Makhlouf HR, Ishak KG (2002) Sclerosed hemangioma and sclerosing cavernous hemangioma of the liver: a comparative clinicopathologic and immunohistochemical study with emphasis on the role of mast cells in their histogenesis. Liver 22(1):70-78

15. Choi YJ, Kim KW, Cha EY, Song JS, Yu E, Lee MG (2008) Case report. Sclerosing liver haemangioma with pericapillary smooth muscle proliferation: atypical CT and MR findings with pathological correlation. $\mathrm{Br}$ J Radiol 81(966):e162-e165

\section{Publisher's Note}

Springer Nature remains neutral with regard to jurisdictional claims in published maps and institutional affiliations.

\section{Submit your manuscript to a SpringerOpen ${ }^{\circ}$ journal and benefit from:}

- Convenient online submission

- Rigorous peer review

- Open access: articles freely available online

- High visibility within the field

- Retaining the copyright to your article

Submit your next manuscript at $\boldsymbol{\nabla}$ springeropen.com 